\title{
Elementos institucionais e desempenho da logística de uma rede pública de assistência farmacêutica
}

\author{
Regina Célia Nazar Fialho \\ IBMEC / Administração \\ Rio de Janeiro / RJ - Brasil \\ Ricardo S. Martins \\ Universidade Federal de Minas Gerais / Centro de Pós-Graduação e Pesquisa em \\ Administração e Núcleo Interdisciplinar de Pesquisas, Ensino e Extensão em Logística \\ Belo Horizonte / MG - Brasil
}

\begin{abstract}
Este trabalho teve como objetivo investigar a influência de elementos institucionais no desempenho da logística de uma organização pública do Brasil. Por meio de uma abordagem quantitativa, utilizando a modelagem de equações estruturais, este estudo identificou que elementos institucionais de caráter regulativo e cognitivo têm influência sobre o desempenho da rede de distribuição de medicamentos, tendo o arranjo da rede um papel de mediação. Além de contribuir para uma melhor compreensão dos fenômenos associados à gestão de cadeias de suprimentos, tendo por base teorias organizacionais, os resultados da pesquisa contribuem para o entendimento de como sistemas simbólicos socialmente constituídos afetam a alocação de recursos e o desempenho de uma função organizacional.
\end{abstract}

Palavras-chave: redes de instalações; desempenho da logística; neoinstitucionalismo.

Elementos institucionales y desempeño logístico de una red pública de atención farmacéutica Este trabajo tiene como objetivo investigar la influencia de elementos institucionales en el desempeño de la logística de una organización pública del Brasil. A través de un abordaje cuantitativo, este estudio identificó que elementos institucionales de carácter regulatorio y cognitivo tienen influencia sobre el desempeño de la red de distribución de medicamentos, teniendo la organización de la red un papel de mediación. Además de contribuir a una mejor comprensión de los fenómenos asociados a la gestión de la cadena de suministro basado en las teorías de organización, los resultados de la investigación contribuyen a la comprensión de cómo los sistemas simbólicos socialmente constituidas afectan a la asignación de recursos y el desempeño de una función organizativa.

Palabras clave: redes de instituciones; desempeño logístico; neoinstitucionalismo.

DOI: http://dx.doi.org/10.1590/0034-7612146817

Artigo recebido em 5 mar. 2015 e aceito em 2 set. 2016 
The Institutional elements and logistics performance of a public pharmaceutical care network This purpose of this paper was to investigate the influence of institutional elements on the logistics performance of a Brazilian public enterprise. By taking a quantitative approach, using structural equation modeling (SEM), this study found that certain institutional elements of a regulative and cognitive nature do have an influence on the performance of the pharmaceutical drug distribution network, with this network arrangement playing a mediating role. In addition to contributing to a better understanding of the phenomena associated with supply chain management, based on organizational theories, the results of this research also contribute to the understanding of how socially constituted symbolic systems can affect the allocation of resources and the performance of an organizational function.

KEYWORDS: facilities networks; logistics performance; neo-institutionalism.

\section{Introdução}

Organizações de serviço público operam em ambientes técnicos fracos e ambientes institucionais fortes, uma vez que dependem da opinião pública para garantir sua legitimidade e precisam estar em conformidade com práticas institucionalizadas (Casile e Davis-Blake, 2002; Meyer e Scott, 1983). Dessa forma, organizações do setor público adotam sistemas de medição de desempenho com uma abordagem orientada por seus valores simbólicos e para atender às expectativas das partes externas interessadas (Roy e Seguin, 2000; Boland e Fowler, 2000).

Assim, considerando fatores contextuais, o desempenho da logística de organizações públicas pode ser explicado a partir de pressões ambientais de caráter institucional, na medida em que tais fatores afetam as escolhas estratégicas de uma cadeia de suprimentos em termos de recursos, estrutura e capacidades (Miemczyk, 2008; Hurley e Hult, 1998).

Recursos podem ser entendidos como elementos de entrada que são controlados e utilizados pelas empresas para desenvolver e implementar suas estratégias. A alocação desses elementos é influenciada pelo contexto institucional afetando o potencial econômico das empresas (Oliver, 1997). Por exemplo: as decisões sobre o projeto de rede de instalações estão relacionadas com localização de instalações, alocação de capacidades e fluxos em uma rede logística. Essas decisões incluem identificação de locais, papéis e capacidades, assim como alocação de mercados/clientes/público que as instalações atenderão (Simchi-Levi, Kaminsky e Simchi-Levi, 2003), ou seja, essas decisões afetam o desempenho da sua função logística (Chopra e Meindl, 2001). Mas como essas decisões são afetadas pelas forças ambientais? Essa pergunta de pesquisa orientou o presente estudo.

O objetivo deste artigo foi identificar a influência de fatores institucionais na configuração e na alocação de recursos de uma rede de instalações pública, de modo a explicar o desempenho de sua logística. Tomando-se por base a política nacional de assistência farmacêutica - um dos alicerces para o desenvolvimento sustentável de políticas públicas de saúde no Brasil —, em síntese, este estudo propôs-se aprofundar a compreensão da influência de fatores institucionais no desempenho e desenho de uma rede pública de distribuição de medicamentos. Dessa forma, este estudo contribui para a literatura de performance em cadeias 
de suprimentos de organizações públicas, delimitando as especificidades da gestão dominada por critérios institucionais, uma vez que esta ainda é pouco explorada (Cai, Jun e Yang, 2010), além de elucidar a importância da decisão de alocação de recursos e a configuração em uma rede de instalações para o desempenho da logística.

Considerando objetivos enunciados, este artigo está estruturado como segue: após esta seção, primeiro é apresentada uma referência teórica com a proposição de um modelo conceitual de pesquisa, para em seguida apresentar a metodologia de pesquisa, incluindo os procedimentos de coleta de dados, mensuração de construtos, teste de hipóteses e resultados. Logo após são apresentadas as discussões e implicações dos resultados, bem como conclusão do trabalho.

\section{Revisão teórica e hipóteses}

Conforme os autores neoinstitucionalistas, as organizações são influenciadas pelas suas redes e contextos (Meyer e Rowan, 1977; Zucker, 1977; Scott, 2008; Greenwood et al., 2008), e o ambiente organizacional é socialmente construído. As ações organizacionais são baseadas em significados compartilhados e suas estruturas se tornam impregnadas de valores que vão além de requisitos técnicos da tarefa (Weick, 1969; Selznick, 1957). Segundo Scott (2008), os estímulos ambientais são cognitivamente processados pelos atores, interpretados pelos indivíduos por meio de sistemas simbólicos socialmente constituídos que, por sua vez, dão origem a instituições ou estruturas sociais que obtêm um alto grau de aceitação.

As instituições são constituídas por elementos analíticos denominados pilares regulativos, normativos e cognitivos. Todos são importantes e atuam em conjunto a partir de processos distintos. Eles postulam diferentes bases de ordem e de conformidade, variando em mecanismos, lógicas e racionalidades para o estabelecimento de reivindicações de legitimidade (Scott, 2008). O pilar regulativo trata do sistema estável de regras, formais e informais, fundamentado em vigilância e poder de sanção. Trata-se de explicitações de forças exteriores que têm por objetivo manter a ordem e mudar o comportamento. A regra pode atuar na institucionalização de comportamentos, podendo, ao longo do tempo, apresentar-se como base de reforço aos valores e normas socialmente aceitos (Scott, 2008). O pilar normativo define normas como sendo a maneira como as coisas deveriam ser realizadas. Ele está relacionado com valores e normas de dimensão prescritiva, de obrigatoriedade do contexto social e organizacional, propiciando a ordem e estabilidade à sociedade. Fundamenta-se na dimensão moral, na qual a preocupação básica é comportamento adequado. Sua lógica é a da adequação e da obrigação ou comportamento moralmente governado, e sua submissão está baseada em expectativas (Scott, 2008). Já o pilar cultural ou cognitivo é o conjunto de conhecimentos e de significados cultural e socialmente criados, empregados como parâmetro de conduta. Salienta o papel central da construção social da realidade mediada por uma estrutura comum de significado (Scott, 2008). 
As instituições são constituídas de várias combinações desses elementos, que variam entre si e, ao longo do tempo, alguns são dominantes. Os elementos institucionais regulativos, normativos e cultural-cognitivos podem estar alinhados ou podem atenuar os efeitos um do outro (Scott, 2005). Para Jackson (2010), esses elementos também podem influenciar um ao outro de maneiras diferentes ao longo do tempo, existindo, assim, um comportamento dinâmico entre os pilares institucionais. Sistemas regulativos, que dependem mais de fiscalização e controles externos, são mais propensos a provocar respostas estratégicas. Elementos normativos, que confiam mais em processos de internalização, são menos propensos a induzir a ações cerimoniais ou respostas resistentes. Já para os elementos cultural-cognitivos, que repousam sobre crenças e suposições, as respostas estratégicas são quase que impossíveis (Tolbert e Zucker, 1999).

O desempenho da logística está relacionado com a mensuração de performance da gestão de uma cadeia de suprimentos (Bowersox, Closs e Cooper, 2002). Ao considerar pressões do ambiente, as regras, normas, valores e crenças têm papel significativo no desempenho de uma cadeia de suprimentos (Hult, Ketchen e Nichols, 2002; Hurley e Hult, 1998; Liang et al., 2007). Quando as metas e os valores são compartilhados por membros da cadeia de suprimentos, as interações contínuas resultam em um processo contínuo e de autorreforço construindo um entendimento compartilhado (Liang et al., 2007; Hult, Ketchen e Arrfelt, 2007; Barney, 1991). Valores, crenças e significados compartilhados em uma cadeia de suprimentos podem explicar o desempenho da empresa em termos de custo, qualidade, entrega e flexibilidade (Krause, Handfield e Tyler, 2007). Porém, nem todas as pressões institucionais influenciam as práticas em gestão de cadeia de suprimentos da mesma forma (Zhu e Sarkis, 2007). Pressões institucionais específicas podem direcionar práticas em gestão de cadeias de suprimentos, bem como seus resultados de forma específica, pois existe uma heterogeneidade de pressões e suas influências.

Nesse direcionamento, um aspecto importante diz respeito à tomada de decisão sobre recursos. Trata-se de um processo normativamente racional que vai de encontro às tradições do ambiente institucional. Embora endógenas, são vulneráveis a pressões institucionais (Oliver, 1997). Os gestores fazem escolhas racionais delimitadas pela incerteza, limitações de informação e por julgamento social (Dimaggio e Powell, 1983; Powell, 1996). Assim, o contexto social afeta a probabilidade de utilização ótima de recursos. No caso específico da gestão de cadeia de suprimentos, o alinhamento de alocação de recursos com elementos institucionais pode desempenhar um papel importante no desempenho da logística de uma organização (He, Brouthers e Filatotchev, 2013). Uma das decisões de alocação de recursos para viabilizar uma função de distribuição e logística diz respeito à configuração e ao desenho da rede de instalações.

O desenho da uma rede de instalações compreende decisões sobre número, localização de instalações de produção e/ou armazenagem, capacidade de cada instalação e conexões entre instalações. Envolve assuntos relacionados com o número de armazéns e plantas, o tamanho dessas instalações, sua localização geográfica, os fluxos físicos, os fluxos de informação necessários e os padrões de fornecimento (Dornier et al., 1998; Meixell e Gargeya, 2005). 
As decisões de projeto de rede de instalações devem estar alinhadas com a configuração definida para o projeto de distribuição adotado pela organização (Chopra, 2003; Simchi-Levi, Kaminsky e Simchi-Levi, 2003), pois, conforme Meepetchdee e Shah (2007), definem restrições para outras decisões táticas e operacionais de uma cadeia de suprimentos, afetando o tempo de resposta, a variedade de produtos, a disponibilidade de produtos, a experiência do cliente e a visibilidade do pedido (Chopra, 2003).

Porém, a configuração da rede de instalações de uma cadeia de suprimentos precisa também considerar as externalidades e o ambiente no qual está inserida, tendo em vista que elementos institucionais de ambientes de cadeias de suprimentos desempenham um papel fundamental na alocação de seus recursos (Zhang e Dhaliwal, 2009; Brouthers, Brouthers e Werner, 2008; Oliver,1997).

\subsection{Hipóteses da pesquisa}

Os recursos públicos precisam ser mais bem planejados e gerenciados, o que quer dizer que há demanda para uma melhor forma de obtenção, aplicação e gestão, como discutem Mesquita e Martins (2008). A administração pública gerencial insere-se num contexto de redefinição da organização do Estado e de suas funções, bem como da ampliação dos instrumentos de controle social e responsabilização (Costa, 2012), o que implica a apropriação e posterior adaptação dos melhores modelos originariamente privados, no sentido de reconstruir a capacidade de gestão do aparelho do Estado.

Na saúde, esse processo tem tido alcance global, com forte expansão da rede de organizações relacionadas com a saúde, que colocam o papel social da saúde dentro dos objetivos de promoção do desenvolvimento (Inoue e Drori, 2006). A mudança institucional ocorrida nos últimos anos na área da saúde trouxe impacto para as formas organizacionais e nos papéis profissionais (Martin, Currie e Finn, 2009). Essa mudança institucional promoveu alterações no atendimento ao paciente, no que diz respeito ao uso de recursos; na padronização do atendimento ao paciente; e na diminuição de variação geográfica quanto à qualidade do serviço prestado (Oakley, 2007).

Com essa perspectiva, a Política Nacional de Medicamentos no Brasil, aprovada em outubro de 1998, tornou-se o instrumento norteador de ações de política de medicamentos. Definiu diretrizes e prioridades, merecendo destaque na reorientação do modelo de assistência farmacêutica, que preconiza a descentralização da gestão; a promoção do uso racional dos medicamentos; a otimização e a eficácia do sistema de distribuição no setor público; e o desenvolvimento de iniciativas que possibilitem a redução nos preços dos produtos (Brasil, 1998). Tendo por base essas diretrizes, o estado de Minas Gerais criou, em 2006, o Programa Farmácia de Minas, com o objetivo de oferecer o serviço de assistência farmacêutica nos diferentes níveis de atenção à saúde, a partir de unidades públicas exclusivas para a distribuição de medicamentos. O programa contou com uma rede de 583 unidades de atendimento para entrega de medicamentos, sendo 154 tipos de medicamentos. 
O programa visou a implantação de farmácias comunitárias públicas nos municípios do estado de Minas Gerais, por meio de incentivo financeiro para o custeio das unidades, ficando as mesmas a cargo da dispensação de medicamentos. Além de se organizar sob a lógica de rede, seria necessário, sobretudo, disponibilizar um serviço de saúde capaz de garantir a acessibilidade da população e ao mesmo tempo obter um desempenho de provisão e recebimento dos medicamentos, propiciando às pessoas a disponibilidade do medicamento para o uso de forma racional. Para tanto, a função logística assumiu um papel fundamental.

Ao considerar o aporte teórico exposto, bem como o contexto analisado, este estudo propõe o modelo conceitual apresentado na figura 1, com base em cinco construtos: Sistema de Regras, Normas e Valores e Crenças (denominados pilares institucionais regulativos, normativos e cognitivos, respectivamente), Desenho da Rede de Instalações e Desempenho da Logística da distribuição de medicamentos básicos do Programa Farmácia de Minas.

Como as bases institucionais do Programa Farmácia de Minas preconizam a eficiência da distribuição logística, os pilares institucionais existentes no programa podem afetar positivamente o desempenho da distribuição de medicamentos básicos. Assim, a conformação dos farmacêuticos responsáveis das farmácias comunitárias às práticas instituídas no programa, sejam essas de ordem regulativa, normativa ou cognitiva, podem afetar positivamente o resultado da performance da logística, bem como o resultado das decisões relativas ao desenho da rede, a partir de uma configuração adequada ao desempenho.

Figura 1

Modelo conceitual proposto

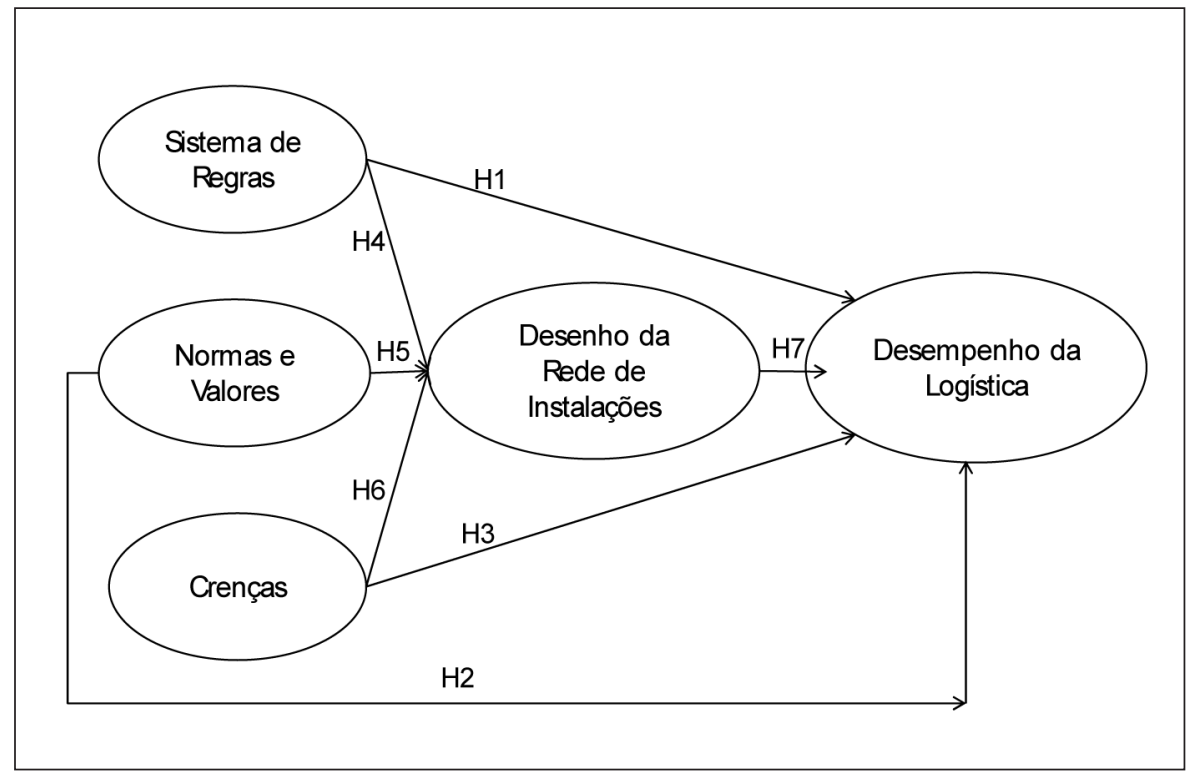

Fonte: Elaborada pelos autores. 
O Pilar Regulativo na área da saúde diz respeito a todas as regras monitoradas e regulamentadas pelo Estado e associações do campo (Caronna, 2004). O Pilar Normativo na área da saúde diz respeito a padrões profissionais de cuidados de saúde, relações esperadas entre profissionais e outros tipos de provedores, e o valor da acessibilidade na prestação de cuidados de alta qualidade (Caronna, 2004). E o Pilar Cognitivo na área da saúde consiste em modelos de cuidados de saúde e crenças (Caronna, 2004). Assim, propõem-se as seguintes hipóteses:

H1: O Sistema de Regras do Programa Farmácia de Minas afeta positivamente o desempenho da logística de distribuição de medicamentos da rede de farmácias comunitárias do Programa.

H2: As normas e os valores do Programa Farmácia de Minas afetam positivamente o desempenho da logística de distribuição de medicamentos da rede de farmácias comunitárias do Programa.

H3: As crenças presentes no Programa Farmácia de Minas afetam positivamente o desempenho da logística de distribuição de medicamentos da rede de farmácias comunitárias do Programa.

H4: O Sistema de Regras do Programa Farmácia de Minas afeta positivamente o desenho da rede de instalações para distribuição de medicamentos.

H5: As normas e os valores do Programa Farmácia de Minas afetam positivamente o desenho da rede de instalações para distribuição de medicamentos.

H6: As crenças presentes no Programa Farmácia de Minas afetam positivamente o desenho da rede de instalações para distribuição de medicamentos.

O desenho e a configuração de uma rede de instalações se referem às decisões de alocação de recursos de modo a suportar o processo de distribuição. Elas definem o número, o tamanho e a localizacão geográfica das unidades que irão compor a rede, bem como aspectos relacionados com o estoques, transporte, fluxos físicos e informacionais e relacionamentos com fornecedores (Dornier et al., 1998; Chopra e Meindl, 2001; Meepetchdee e Shah, 2007). O conjunto dessas decisões pode afetar o resultado da performance logística. Essas decisões também sofrem influência de fatores institucionais (Brouthers, Brouthers e Werner, 2008; Oliver, 1997) e podem atuar como mediadores no relacionamento entre os elementos institucionais e o desempenho da logística de distribuição de uma rede de farmácias comunitárias. A partir do exposto, propõe-se:

H7: O desenho da rede de instalações do Programa Farmácia de Minas afeta positivamente o desempenho da logística de distribuição de medicamentos da rede de farmácias comunitárias do Programa.

Uma vez apresentado o modelo conceitual proposto para a pesquisa, a seguir são apresentados todos os aspectos metodológicos utilizados para a concretização do objetivo do estudo. 


\section{Procedimentos metodológicos}

\subsection{Unidade de análise}

A unidade de análise deste estudo são as práticas institucionalizadas no Programa Farmácia de Minas, voltadas para a recuperação da saúde do estado de Minas Gerais, e seu impacto no desempenho da logística da rede de instalações de distribuição de medicamentos do componente básico.

\subsection{Coleta de dados}

Para a fase qualitativa, este estudo realizou levantamentos por mala direta mediante entrevistas com 15 farmacêuticos responsáveis pelas farmácias comunitárias e quatro gerentes da Secretaria de Saúde de Minas envolvidos no processo de distribuição de medicamentos do Programa Farmácia de Minas, para obtenção e definição de quais variáveis comporiam os construtos relativos a pilares institucionais. As variáveis obtidas foram validadas vis-à-vis sua elaboração teórica por um especialista em estudos organizacionais.

As variáveis relativas aos construtos Desenho da Rede de Instalações e Desempenho foram identificadas a partir do aporte teórico previamente pesquisado e com base na disponibilidade de informações da Subsecretaria de Inovação e Logística do Estado de Minas Gerais.

Para a fase quantitativa e avaliação da conformação às instituições presentes no Programa Farmácia de Minas, foram realizadas 150 entrevistas por telefone com os farmacêuticos responsáveis pelas respectivas farmácias, definidas por amostragem aleatória simples. Foi utilizado um cross-sectional survey com questões valoradas em escala Likert.

\subsection{Desenvolvimento de instrumentos e medidas}

A partir dos construtos definidos no modelo conceitual, foram identificadas 30 variáveis de mensuração. Para análise da estrutura fatorial das variáveis relativas aos pilares institucionais, que compõem os primeiros três construtos caracterizados como reflexivos, foi verificada sua dimensionalidade, a partir da Análise Fatorial Exploratória, e em seguida sua fidedignidade, a partir da Validade Convergente, Discriminante e Confiabilidade obtidas pela Análise Fatorial Confirmatória.

As medidas estimadas pela Análise Fatorial Exploratória foram obtidas pelo método de Componentes Principais, com rotação Varimax. A determinação dos fatores subjacentes ao conjunto de dados foi realizada pelo critério da percentagem de variância explicada superior a $60 \%$, uma vez que se está perante um fenômeno alusivo às ciências sociais (Hair et al., 2009). Ainda foram identificados e removidos os itens com communalities inferiores a 0.40 
com factor loadings inferiores a 0.40, uma vez que se trata de uma pesquisa exploratória (Hair et al., 2009).

Foram ainda determinadas as medidas Kaiser-Meyer-Olkin (KMO) para avaliar a adequabilidade da utilização da análise fatorial. Assim, a análise da estrutura relacional identificou uma solução final composta por 16 itens e cinco fatores que explicam 61,09\% da variância da escala (anexo I). Essa solução fatorial apresenta um KMO de 0.822 e o teste de esfericidade de Bartlett apresenta valores adequados $\left(\chi^{2}(406)=664.52 ; \mathrm{p}<0.001\right)$.

Em relação às variáveis pertencentes aos construtos Desenho da Rede de Instalações e Desempenho da Logística, construtos caracterizados como formativos, foi realizada Análise de Multicolinearidade e Validade Convergente dos mesmos. Observando os valores de Inflacionamento da Variância das Variáveis (VIF) do construto Desenho da Rede e do construto Desempenho da Logística, observou-se que nenhuma delas apresentou um valor acima de 3.3, sendo possível concluir que não existem indícios de multicolinearidade que poderiam afetar os resultados.

Entretanto, no caso do construto Desenho da Rede, duas variáveis não apresentaram validade convergente, bem como uma variável do construto Desempenho da Logística. Todavia, optou-se por mantê-las, uma vez que a teoria utilizada é capaz de suportar a permanência de tais variáveis, somando-se ao fato de que o efeito de sua retirada era praticamente nulo (Hair et al., 2013). Diante desses resultados, verificou-se que os construtos possuem Validade Convergente e Validade Discriminante, bem como níveis de confiabilidade aceitáveis para verificação de sua validade nomológica.

\section{Resultados}

Para a estimação do modelo estrutural, e avaliação das respetivas hipóteses, foi utilizado o software SmartPLS 2.0 (Christian Ringle, Sven Wende e Alexander Will, Germany), com estimação por meio do método dos mínimos quadrados parciais (Partial Least Squares -PLS), uma vez que a dimensão da amostra $(n=150)$ era reduzida para efetuar a estimação pelos modelos baseados na covariância, e os resultados mediante o método PLS são mais robustos, com menos problemas de identificação, em amostras de menores dimensões (Hair et al., 2011). Para determinação das estatísticas $t$ e respectiva significância estatística recorreu-se à metodologia de bootstrapping com 200 réplicas de amostras constituídas por 128 casos.

Em termos de modelagem de equações estruturais, foram avaliados dois modelos conceituais. Um primeiro em que o construto alusivo ao Desenho da Rede possui um efeito mediador na relação entre os Pilares Institucionais e o Desempenho da logística, apresentado na figura 2 e na tabela 1, e um segundo modelo em que os Pilares Institucionais exercem um efeito direto sobre o Desempenho da Logística, como demonstrado na figura 3 e na tabela 2. 
Figura 2

Modelo com mediação

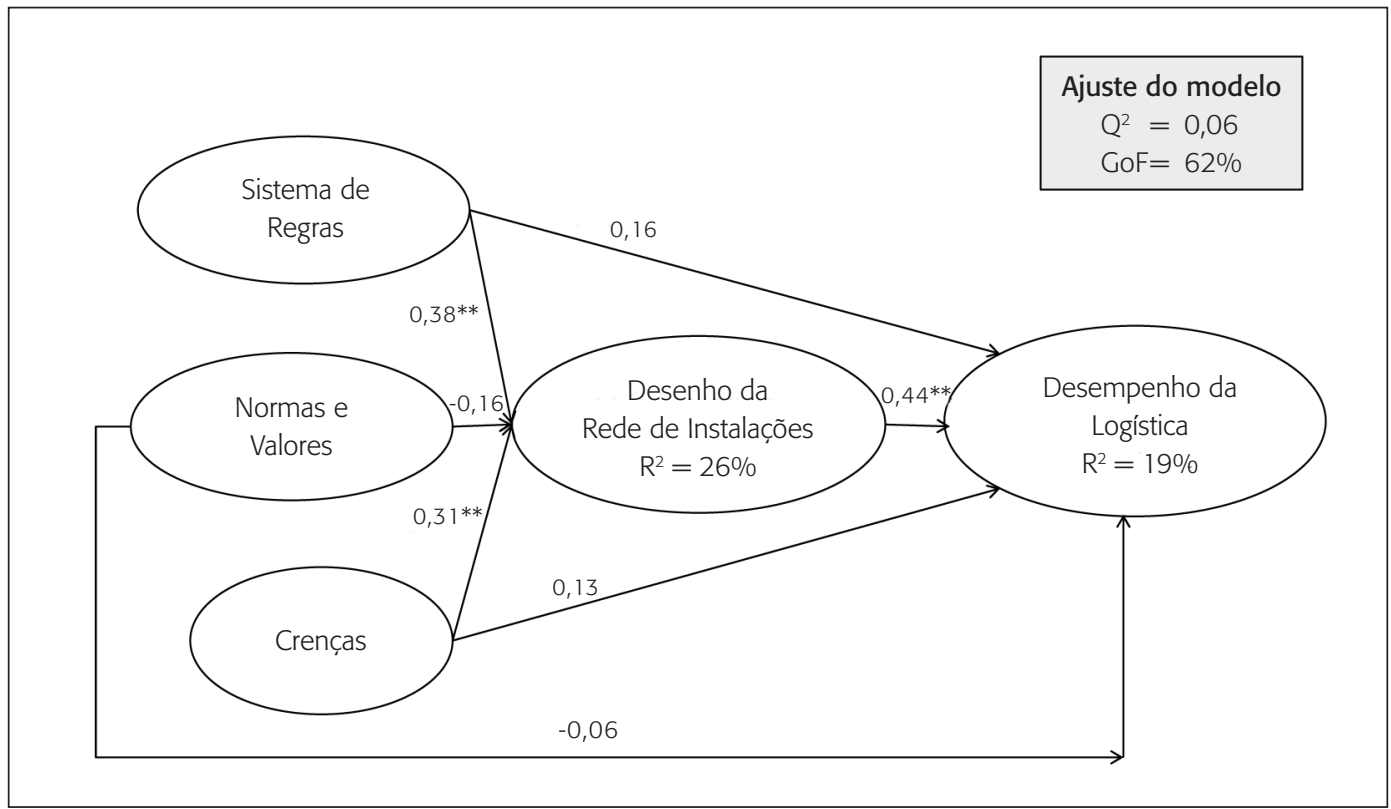

Fonte: Elaborada pelos autores.

Tabela 1

Resultado do teste de hipótese e R2 para o modelo com mediação

\begin{tabular}{|llcrr|}
\hline Construto & & Carga & Valor t & Sig. \\
\hline Exógeno & Endógeno & & & \\
\hline 1. Sistema de Regras & 4. Desenho da Rede & 0,38 & 2,17 & $1,56 \%$ \\
2. Normas e Valores & $\mathrm{R}^{2}=26 \%$ & $-0,16$ & 0,89 & $18,51 \%$ \\
3. Crenças & & 0,31 & 2,35 & $1,0 \%$ \\
\hline 1. Sistema de Regras & & 0,16 & 1,70 & $4,52 \%$ \\
2. Normas e Valores & 5. Desempenho da Logística & $-0,06$ & 0,73 & $23,06 \%$ \\
3. Crenças & $\mathrm{R}^{2}=19 \%$ & 0,13 & 1,89 & $2,97 \%$ \\
4. Desenho da rede & & 0,44 & 6,26 & $0,00 \%$ \\
\hline
\end{tabular}

Fonte: Resultados da pesquisa.

Uma vez que não existem medidas de bondade de ajuste global adequadas para os modelos estimados com PLS como nas metodologias de equações estruturais baseadas nas covariâncias, os modelos estruturais estimados por PLS foram avaliados por meio da análise dos valores de $\mathrm{R}^{2}$ para os construtos endógenos (Henseler e Fassott, 2010; Henseler, Ringle e Sinkovics, 2009). Segundo Cohen (1988), valores de $\mathrm{R}^{2}$ igual ou superior a $26 \%$ representam 
um efeito grande. Assim, os resultados apontam que os construtos referentes aos elementos institucionais exercem efeito grande sobre a alocação de recursos da rede de instalações. Já a alocação de recursos do desenho da rede de instalações exerce efeito médio sobre o Desempenho da Logística de distribuição de medicamentos do Programa Farmácia de Minas.

Figura 3

Modelo com efeito direto sobre o Desempenho da Logística

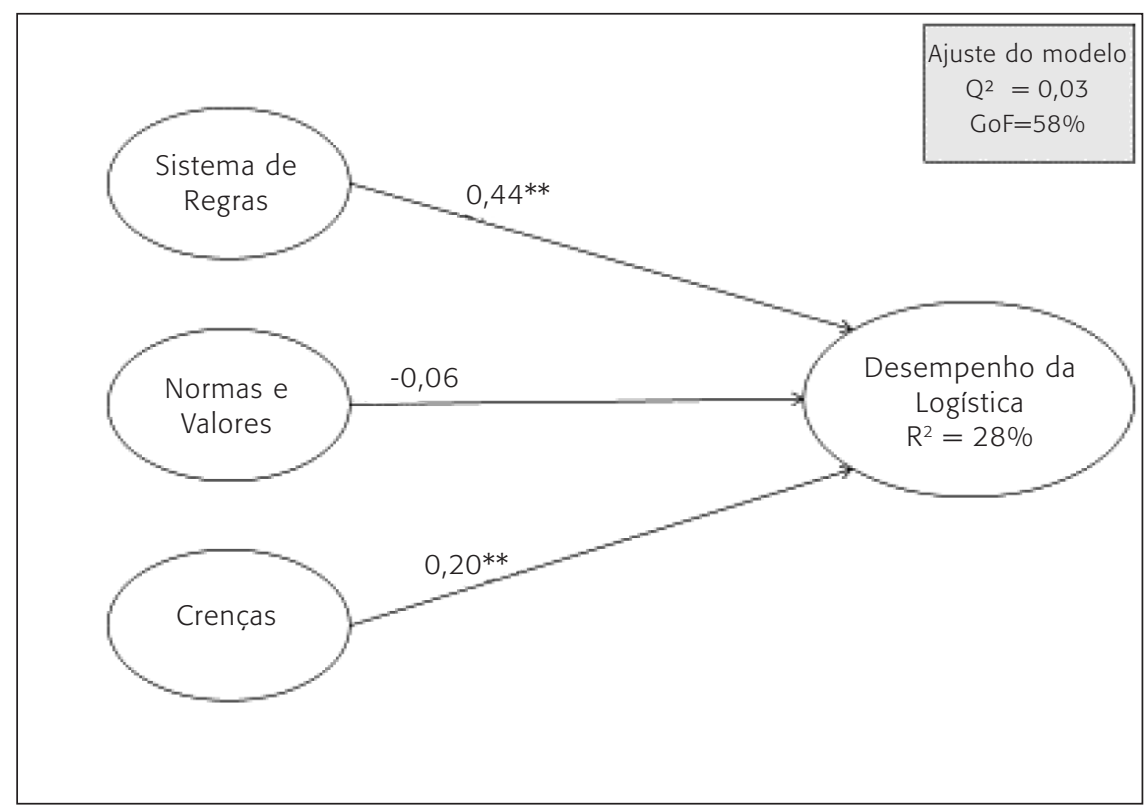

Fonte: Elaborada pelos autores.

Tabela 2

Resultado do teste de hipótese e R2 para o modelo sem mediação

\begin{tabular}{|llrrr|}
\hline & Construto & Carga & Valor t & Sig. \\
\cline { 1 - 2 } Exógeno & Endógeno & & & \\
\hline 1. Sistema de Regras & & 0,44 & 3,04 & $0,14 \%$ \\
2. Normas e Valores & 5. Desempenho & $-0,06$ & 0,24 & $40,4 \%$ \\
3. Crenças & R $^{2}=28 \%$ & 0,20 & 1,63 & $1,05 \%$ \\
\hline
\end{tabular}

Fonte: Resultados da pesquisa.

A partir dos dados apresentados nas tabelas 1 e 2, percebe-se que houve alteração significativa nas cargas dos construtos Sistema de Regras e Crenças, em relação ao Desempenho da Logística, que, com a presença do construto Desenho da Rede, não eram significativas. Dessa forma, percebe-se que o construto Desenho da Rede absorve parte do efeito desses dois cons- 
trutos, caracterizando-se como construto mediador no relacionamento entre os construtos referentes aos pilares institucionais e o construto Desempenho da Logística (Abbad e Torres, 2002).

Para avaliar a qualidade de ajuste do modelo estrutural, foram utilizadas as medidas de validade preditiva ou Stone-Geisser's $\left(Q^{2}\right)$ e o GoF (Goodness of Fit). O indicador de StoneGeisser $Q^{2}$ foi identificado no módulo Blindfolding do SmartPLS pela leitura da redundância geral do modelo (Ringle; Da Silva e Bido, 2014). Já o GoF foi obtido pela média geométrica entre o $\mathrm{R}^{2}$ médio (adequação do modelo estrutural) e a média ponderada das AVE (adequação do modelo de mensuração). Esse índice pode variar de 0\% a 100\%, e Wetzels, OdekerkenSchroder e Van Oppen (2009) sugerem valores acima de 0,36 como adequados para as áreas de ciências sociais e do comportamento (Ringle, Da Silva e Bido, 2014). A tabela 3 demostra os valores encontrados - GoF e $\mathrm{Q}^{2}$ - dos construtos endógenos do modelo.

Tabela 3

Valores Gof e Q2

\begin{tabular}{|lcccccc|}
\hline \multirow{2}{*}{$\begin{array}{l}\text { Medidas de } \\
\text { Ajuste }\end{array}$} & \multicolumn{2}{c}{$\mathrm{R}^{2}$} & \multicolumn{2}{c}{ GoF } & \multicolumn{2}{c|}{$\mathrm{Q}^{2}$} \\
\cline { 2 - 7 } & Efeito direto & Mediação & Efeito direto & Mediação & Efeito direto & Mediação \\
\hline Desenho da Rede & - & $26 \%$ & \multirow{2}{*}{$58 \%$} & $62 \%$ & - & 0,05 \\
Desempenho & $28 \%$ & $19 \%$ & & & 0,03 & 0,06 \\
\hline
\end{tabular}

Fonte: Resultados da pesquisa.

A partir dos dados encontrados, os indicadores de ajustes apontam que o modelo com o construto Desenho da Rede se mostra mais adequado, com um valor maior de predição. Assim, as hipóteses H4, H6 e H7 foram aceitas. As demais hipóteses não foram comprovadas pelo método estatístico aplicado.

\section{Discussões e implicações}

Com base nos dados levantados na fase qualitativa da pesquisa, a partir do novo paradigma vigente na área de saúde e de assistência farmacêutica, constatou-se que os responsáveis pelas unidades de atendimento percebem como regras do Programa Farmácia de Minas atividades referentes à programação, ao recebimento, ao manuseio e à entrega dos medicamentos ao paciente. Além disso, a função de orientação ao paciente quanto ao uso e manutenção do medicamento também é percebida como regra.

Já os valores e normas de dimensão prescritiva, vistos como maneiras adequadas e justas, foram percebidos como sendo o atendimento a todos de forma indistinta, a entrega de medicamentos prioritária às unidades com Índice de Desenvolvimento Humano (IDH) menores, a quantidade de medicamentos disponíveis a partir do número de habitantes do município, e a estrutura física exclusiva e próxima ao posto de saúde como importantes para o atendimento eficaz ao paciente. 
No que diz respeito às crenças e aos significados compartilhados no Programa Farmácia de Minas, segundo os responsáveis pelas unidades de atendimento do Programa Farmácia de Minas, foram mencionadas as crenças de que a farmácia comunitária é vista como local onde as ações de assistência farmacêutica podem se concretizar, onde o acompanhamento farmacoterapêutico é fundamental para a melhoria de qualidade de vida da população, e a crença de que o Programa Farmácia de Minas é referência no país.

Com base nessas informações e na construção do modelo estrutural e de mensuração, foi possível identificar que, ao considerar os efeitos diretos dos aspectos institucionais no desempenho da logística, constatou-se que existe um efeito significativo dos componentes regulativos e cognitivos. O sistema de regras e as crenças compartilhadas no Programa Farmácia de Minas afetam de forma significativa o desempenho da logística de distribuição de medicamentos, em termos de tempo de entrega dos medicamentos, percentual de uso da capacidade instalada disponível para estocar medicamentos, tempo em que a farmácia comunitária fica sem estoque, custo de transporte para entrega de medicamentos, percentual de exatidão das informações relativas a demanda e estoque, e percentual de medicamentos faltantes devido a atrasos de fornecedores.

Quanto mais os farmacêuticos responsáveis pelas farmácias comunitárias se adequam às práticas institucionalizadas de caráter regulativo e cognitivo, maior é o desempenho da logística, enquanto conjunto de indicadores relacionados com a entrega do medicamento, estoque e uso da capacidade instalada. Já o mesmo não foi constatado para as práticas institucionalizadas de caráter normativo. Ou seja, a adequação dos responsáveis pelas farmácias comunitárias ao percebido como maneiras adequadas para se realizar algo não afetam o desempenho da logística de distribuição de medicamentos.

Segundo os neoinstitucionalistas, os elementos institucionais podem estar alinhados e se reforçarem mutuamente, ou podem conter ambiguidades, gerando efeitos econômicos adversos (Crouch, 2005; Deeg e Jackson, 2007). Ademais, sistemas regulativos são mais propensos a provocar respostas cerimoniais ou estratégicas (Tolbert e Zucker, 1999).

Assim, para o caso do Programa Farmácia de Minas, percebe-se um alinhamento entre os elementos institucionais regulativos e cogntivos de modo a afetar o desempenho da logística de distribuição de medicamentos. As regras relativas ao ciclo de assistência farmacêtica associadas ao significado cultural do que é o programa estão alinhadas ao desempenho de uma série de indicadores de desempenho da logística de distribuição de medicamentos. E mesmo que os aspectos regulativos do programa possam ser passíveis de uma conformação cerimonial, pois aqui o instrumento de análise são questionários respondidos, os elementos cognitivos ainda atuam no sentido de influenciar o desempenho da logística.

Já no que diz respeito à alocação de recursos, como proposto por alguns autores, o ambiente institucional influencia decisões de alocação de capacidades e de recursos (Hurley e Hult, 1998; Zhang e Dhaliwal, 2009; Brouthers, Brouthers e Werner, 2008; Oliver, 1997). Com base nessa perspectiva e nos resultados apontados pelo modelo estrutural e de mensuração, ficou constatado que os elementos institucionais do Programa Farmácia de Minas afetam o resultado da configuração da rede de instalações da logística de distribuição em termos de 
capacidade da rede e configuração de fluxo. E esse efeito ocorre de maneira diferenciada entre os elementos institucionais.

Assim, quanto mais as unidades de atendimento do Programa Farmácia de Minas se conformam às regras de otimização das atividades de programação, recebimento, manuseio, dispensação dos medicamentos, e orientação ao paciente quanto ao uso racional do medicamento, bem como crenças relativas ao programa, maior é o efeito sobre a configuração do Desenho da Rede de instalações. Assim, o sistema de regras e crenças presentes no Programa Farmácia de Minas afeta a localização e o tamanho das instalações das unidades de atendimento, assim como capacidades de estoques alocados a cada unidade, volumes de transporte necessários, nível de utilidade de informações de demanda e estoques e número de fornecedores necessários para suprir as entregas das unidades de atendimento.

Todavia, para o caso de Normas e Valores presentes no Programa, a amplitude no atendimento às pessoas, a prioridade na entrega às unidades com base ao IDH, a quantidade de medicamentos disponíveis a partir do número de habitantes do município, e a importância da estrutura física exclusiva e a proximidade do posto de saúde têm efeito inverso e não significativo sobre a alocação de capacidades e fluxos na rede de distribuição de medicamentos. Os valores e normas considerados apropriados e adequados para a assistência farmacêutica demonstram nenhuma influência sobre a configuração da rede de instalações, por apresentar importância menor ante as vantagens econômicas dos recursos disponíveis para esse quesito (Brouthers, Brouthers e Werner, 2008).

Assim, existe um efeito direto entre a conformação dos responsáveis pelas farmácias comunitárias às práticas institucionalizadas de caráter regulativo e cognitivo com a abrangência, a capacidade, o fluxo e a adequação da rede de instalações como recurso da função logística.

No que diz respeito ao modelo conceitual proposto, indicado neste estudo, embora exista um efeito direto dos pilares institucionais no desempenho da logística de distribuição, o modelo com maior valor preditivo apresenta a decisão de configuração da rede de instalações como construto mediador no relacionamento. Assim, a decisão quanto à configuração da rede de instalações ajuda a explicar o efeito do ambiente institucional no desempenho da logística de distribuição.

A configuração das instalações enquanto capacidade, fluxo e abrangência se mostrou a variável que transformou e traduziu os elementos institucionais em performance, em uma rede de distribuição de medicamentos pública. Ela se definiu como uma dimensão fundamental a ser tratada, diante das especificidades de uma rede de distribuição de medicamentos, que precisa ser operacionalizada com um alto grau de pulverização.

Dessa forma, com base no modelo proposto e testado, é possível demonstrar que o ambiente institucional pode influenciar o resultado de uma função organizacional, bem como suas decisões de alocação de capacidades e de recursos (Hurley e Hult, 1998; Huber, 1991; Zhang e Dhaliwal, 2009; Brouthers, Brouthers e Werner, 2008; Oliver, 1997), principalmente em se tratando de organizações públicas (Said e Higgins, 2005), podendo ser considerado um aspecto para obtenção de vantagem competitiva. Ademais, ao se deparar com diagnósticos 
relativos ao desempenho da logística, decisões relativas à configuração de uma rede de suprimento mostram-se com grande poder explicativo.

A necessidade de uma administração pública gerencial decorre de problemas de crescimento e de aumento de complexidade, e também de legitimação da burocracia perante as demandas dos cidadãos (Bresser-Pereira, 1996). A nova gestão pública está atrelada ao desempenho eficiente da tarefa administrativa, relacionada com uma estratégia e com um comportamento organizacional, tendo como pano de fundo o argumento do alinhamento imperativo entre o administrador e as necessidades dos cidadãos, um aspecto onde a eficiência seria a lei imperante na administração pública (Prado, 2010). A adoção do princípio da eficiência pela Constituição Federal faz com que a administração pública não fique simplesmente restrita à prestação de serviços eficazes e pontuais, mas, sim, faz com que ela busque tecnologias e métodos modernos para obter a qualidade total na execução de suas atividades, por exemplo, buscando um novo foco na administração dos serviços e atividades públicas, de meramente fiscalistas, como em tempos atrás, para um foco no desenvolvimento. Desse entendimento, contextualizam-se as justificativas para a adoção de práticas de cadeias de suprimentos para reforma das práticas gerenciais da administração pública brasileira, conforme destacado por Tridapalli, Fernandes e Machado (2011).

\section{Conclusões}

Abordar aspectos referentes ao desempenho da logística em organizações públicas requer uma abordagem mais elaborada, pois os tradicionais objetivos de custos e atendimento ao cliente estão emaranhados em fatores institucionais e ambientais. Cadeias de suprimento que atuam no setor público fazem parte de uma complexa teia de organizações, cujos papéis estão sujeitos a demandas sociais e políticas, tanto da comunidade quanto do governo. Nessa perspectiva, a teoria neoinstitucional pode contribuir para explicar como aspectos sociais atuam sob a estrutura e práticas organizacionais.

No caso deste estudo, foi identificado que o desempenho da logística é mais reativo aos aspectos institucionais relativos aos pilares regulativos e cognitivos. Essa constatação corrobora os neoinstitucionalistas, ao postularem que organizações públicas precisam obter maior legitimidade e, assim, estão mais sujeitas a sofrer pressões de caráter regulativo, além de demonstrar que as influências institucionais nem sempre atuam de forma uniforme. Além disso, é notório o uso de regras e normas reguladoras no sistema de saúde brasileiro como uma alternativa viável para a superação de entraves para o fortalecimento de um sistema de saúde integral e eficiente.

Assim, este estudo oferece subsídios para apoiar a decisão dos gestores de saúde e logística de uma operação pública, no entendimento de quais elementos podem fortalecer suas vantagens em recursos, além de direcionar os esforços dos envolvidos para alcance de seus objetivos. Além disso, contribui para a produção de conhecimento ao demonstrar que fatores de contexto institucional podem ser úteis na explicação do desempenho da logística, além de 
apontar a importância do papel do desenho da rede de instalações no desempenho da área e, consequentemente, na obtenção dos resultados planejados.

Todavia, o presente estudo avaliou qual é o relacionamento entre os fatores institucionais e o desempenho de uma função organizacional, mas não entrou no mérito em avaliar o quanto a institucionalização de práticas impacta o desempenho da saúde como campo de análise. Existem indicadores de saúde que poderiam ter sido confrontados. Além disso, é sabido que o Programa Farmácia de Minas foi alterado por Programa Farmácia de Todos, tendo em vista mudanças de governos de estado. Assim, para futuras pesquisas, sugere-se que o impacto do uso de práticas institucionalizadas seja analisado em um contexto maior, considerando também variáveis relativas a interrupções e trocas de governos, bem como o descompasso de atuação entre as três esferas de governo.

\section{Referências}

ABBAD, Gardênia; TORRES, Cláudio V. Regressão múltipla stepwise e hierárquica em psicologia organizacional: aplicações, problemas e soluções. Estudos de Psicologia, Natal, v. 7, n. esp., p. 19$29,2002$.

BARNEY, Jay. Firm resources and sustained competitive advantage. Journal of Management, v. 17, n. 1, p. 99-120, 1991.

BOLAND, Tony; FOWLER, Alan. A systems perspective of performance management in public sector organizations. International Journal of Public Sector Management, v. 13, n. 5, p. 417-446, 2000.

BOWERSOX, Donald J.; CLOSS, David J.; COOPER, M. Bixby. Gestão logística de cadeias de suprimentos. Tradução de Camila Teixeira Nakagawa e Gabriela Teixeira. Porto Alegre: Bookman, 2002.

BRASIL. Ministério da Saúde. Portaria GM n. 3.916, de 30 out. 1998. Política Nacional de Medicamentos. Diário Oficial [da] República Federativa do Brasil, Poder Executivo, Brasília DF, 10 nov. 1998.

BRESSER-PEREIRA, Luiz Carlos. Da administração pública burocrática à gerencial. Revista do Serviço Público, v. 47, n. 1, p. 7-40, 1996.

BROUTHERS, Keith D.; BROUTHERS, Lance E.; WERNER, Steve. Resource-based advantages in an international context. Journal of Management, v. 34, n. 2, p. 189-217, 2008.

CAI, Shaohan; JUN, Minjoon; YANG, Zhilin. Implementing supply chain information integration in China: the role of institutional forces and trust. Journal of Operations Management, v. 28, n. 3, p. 257-268, 2010.

CARONNA, Carol A. The misalignment of institutional "pillars": consequences for the US health care field. Journal of Health and Social Behavior, v. 45, Extra Issue: Health and Health Care in the United States, p. 45-58, 2004.

CASILE, Maureen; DAVIS-BLAKE, Alison. When accreditation standards change: factors affecting differential responsiveness of public and private organizations. Academy of Management Journal, v. 45, n. 1, p. 180-195, 2002. 
CHOPRA, Sunil. Designing the distribution network in a supply chain. Transportation Research Part E: Logistics and Transportation Review, v. 39, n. 2, p. 123-140, 2003.

CHOPRA, Sunil; MEINDL, Peter. Supply chain management: strategy, planning and operation. Upper Saddle River, NJ: Prentice-Hall. 2001.

COHEN, Morris A.; LEE, Hau L. Strategic analysis of integrated production-distribution systems: models and methods. Operations Research, v. 36, n. 2, p. 216-228, 1988.

COSTA, Gustavo P. Heranças patrimonialistas, (dis)funções burocráticas, práticas gerenciais e os novos arranjos do estado em rede: entendendo a configuração atual da administração pública brasileira. Tese (doutorado em administração) — Escola Brasileira de Administração Pública e de Empresas, Fundação Getulio Vargas, Rio de Janeiro, 2012.

CROUCH, Colin. Capitalist diversity and change: recombinant governance and institutional entrepreneurs. Oxford: Oxford University Press, 2005.

DEEG, Richard; JACKSON, Gregory. Towards a more dynamic theory of capitalist variety. Socio-Economic Review, v. 5, n. 1, p. 149-179, 2007.

DIMAGGIO, Paul J.; POWELL, Walter W. The iron cage revisited: institutional isomorphism and collective rationality in organizational fields. American Sociological Review, v. 48, n. 2, p. 147-160, 1983.

DORNIER, Philippe-Pierre et al. Global operations and logistics: text and cases. Nova York: John Wiley \& Sons, 1998.

GREENWOOD, Royston et al. (Ed.). The Sage handbook of organizational institutionalism. Los Angeles: Sage, 2008.

HAIR, Joseph F. et al. A primer on partial least squares structural equation modeling (PLS-SEM). Thousand Oaks: Sage Publications, Incorporated, 2013.

HAIR, Joseph F. et al. Multivariate data analysis. Upper Saddle River, NJ: Prentice Hall, 2009.

HAIR, Joseph F.; RINGLE, Christian M.; SARSTEDT, Marko. PLS-SEM: indeed a silver bullet. The Journal of Marketing Theory and Practice, v. 19, n. 2, p. 139-152, 2011.

HE, Xinming; BROUTHERS, Keith D.; FILATOTCHEV, Igor. Resource-based and institutional perspectives on export channel selection and export performance. Journal of Management, v. 39, n. 1, p. 27-47, 2013.

HENSELER, Jörg; FASSOTT, Georg. Testing moderating effects in PLS path models: an illustration of available procedures. In: ESPOSITO VINZI, V. et al. (Ed.). Handbook of partial least squares. Heidelberg: Springer-Verlag, 2010. p. 713-735.

HENSELER, Jörg; RINGLE, Christian M.; SINKOVICS, Rudolf R. The use of partial least squares path modeling in international marketing. Advances in International Marketing, v. 20, n. 1, p. 277319, 2009.

HUBER, George P. Organizational learning: the contributing processes and the literatures. Organization Science, v. 2, n. 1, p. 88-115, 1991. 
HULT, G. Tomas M.; KETCHEN, David J.; ARRFELT, Mathias. Strategic supply chain management: improving performance through a culture of competitiveness and knowledge development. Strategic Management Journal, v. 28, n. 10, p. 1035-1052, 2007.

HULT, G. Tomas M.; KETCHEN, David J.; NICHOLS, Ernest L. An examination of cultural competitiveness and order fulfillment cycle time within supply chains. Academy of Management Journal, v. 45, n. 3, p. 577-586, 2002.

HURLEY, Robert F.; HULT, G. Tomas M. Innovation, market orientation, and organizational learning: an integration and empirical examination. The Journal of Marketing, v. 62, n. 3, p. 42-54, 1998.

INOUE, Keiko; DRORI, Gili S. The global institutionalization of health as a social concern organizational and discursive trends. International Sociology, v. 21, n. 2, p. 199-219, 2006.

JACKSON, Gregory. Actors and institutions. The Oxford handbook of comparative institutional analysis. Oxford: Oxford University Press, 2010. p. 63-86.

KRAUSE, Daniel R.; HANDFIELD, Robert B.; TYLER, Beverly B. The relationships between supplier development, commitment, social capital accumulation and performance improvement. Journal of Operations Management, v. 25, n. 2, p. 528-545, 2007.

LIANG, Huigang et al. Assimilation of enterprise systems: the effect of institutional pressures and the mediating role of top management. Management Information Systems Research Center, v. 31, n. 1, p. 59-87, 2007.

MARTIN, Graham P.; CURRIE, Graeme; FINN, Rachael. Reconfiguring or reproducing intra-professional boundaries? Specialist expertise, generalist knowledge and the 'modernization' of the medical workforce. Social Science \& Medicine, v. 68, n. 7, p. 1191-1198, 2009.

MEEPETCHDEE, Yongyut; SHAH, Nilay. Logistical network design with robustness and complexity considerations. International Journal of Physical Distribution \& Logistics Management, v. 37, n. 3, p. 201-222, 2007.

MEIXELL, Mary J.; GARGEYA, Vidyaranya B. Global supply chain design: a literature review and critique. Transportation Research Part E: Logistics and Transportation Review, v. 41, n. 6, p. 531-550, 2005.

MESQUITA, Arlan M.; MARTINS, Ricardo S. Desafios logísticos às redes de negócios no Brasil: o que podem as Parcerias Público-Privadas (PPP)? Rev. Adm. Pública, v. 4, n. 42, p. 735-763, 2008.

MEYER, John W.; ROWAN, Brian. Institutionalized organizations: formal structure as myth and ceremony. American Journal of Sociology, v. 83, n. 2, p. 340-363, 1977.

MEYER, John W.; SCOTT, W. Richard. Organizational environments: ritual and rationality. Beverly Hills, California: Sage, 1983.

MIEMCZYK, Joe. An exploration of institutional constraints on developing end-of-life product recovery capabilities. International Journal of Production Economics, v. 115, n. 2, p. 272-282, 2008.

OAKLEY, Christine. Health care reform, public health and institutional change. Conference Papers - American Sociological Association. Annual Meeting, 2007. p. 33. 
OLIVER, Christine. Sustainable competitive advantage: combining institutional and resource-based views. Strategic Management Journal, v. 18, n. 9, p. 697-713, 1997.

POWELL, Walter W. On the nature of institutional embeddedness: labels vs explanation. Advances in Strategic Management, v. 13, p. 293-300, 1996.

PRADO, Henrique S. A. Nova gestão pública para o desenvolvimento. In: ERKENS, Rainer (Org.). Nova gestão pública. São Paulo: Instituto Friedrich Naumann, 2010. p. 17-25.

RINGLE, Christian M.; DA SILVA, Dirceu; BIDO, Diógenes de Souza. Modelagem de equações estruturais com utilização do Smartpls. Revista Brasileira de Marketing, v. 13, n. 2, p. 54-71, 2014.

ROY, Claude; SEGUIN, Francine. The institutionalization of efficiency-oriented approaches for public service improvement. Public Productivity \& Management Review, v. 12, n. 4, p. 449-468, 2000.

SAID, Taoufik; HIGGINS, Anne. Exploring innovative HRM practices in the public sector: the case of a Canadian health emergency organization. Management Research News, [s. 1.], v. 28, n. 9, p. 35-40, 2005.

SCOTT, W. Richard. Institutional theory: contributing to a theoretical research program. In: SMITH, Ken G.; HITT, Michael A. (Ed.). Great minds in management: the process of theory development. Oxford: Oxford University Press, 2005. p. 460-484.

SCOTT, W. Richard. Institutions and organizations: ideas and interests. Los Angeles, California: Aufl., 2008.

SELZNICK, Philip. Leadership in administration: a sociological interpretation. Evanston, IL: Row, Peterson and Company, 1957.

SIMCHI-LEVI, David; KAMINSKY, Philip; SIMCHI-LEVI, Edith. Cadeia de suprimentos projeto e gestão: conceitos, estratégias e estudos de caso. Porto Alegre: Bookman, 2003.

TOLBERT, Pamela S.; ZUCKER, Lynne G. A institucionalização da teoria institucional. In: CLEGG, Stewart R.; HARDY, Cynthia; NORD, Walter R. Handbook de estudos organizacionais. São Paulo: Atlas, 1999. v. 1, p. 196-219.

TRIDAPALLI, Juarez P.; FERNANDES, Elton; MACHADO, Waltair V. Gestão da cadeia de suprimento do setor público: uma alternativa para controle de gastos correntes no Brasil. Rev. Adm. Pública, Rio de Janeiro, v. 45, n. 2, p. 401-433, mar./abr. 2011.

WEICK, Karl E. The social psychology of organizing. Reading, MA: Addison- Wesley, 1969.

WETZELS, Martin; ODEKERKEN-SCHRODER, Gaby; VAN OPPEN, Claudia. Using PLS path modeling for assessing hierarchical construct models: guidelines and empirical illustration. Management Information Systems Quarterly, v. 33, n. 1, p. 177-195, 2009.

ZHANG, Cheng; DHALIWAL, Jasbir. An investigation of resource-based and institutional theoretic factors in technology adoption for operations and supply chain management. International Journal of Production Economics, v. 120, n. 1, p. 252-269, 2009. 
ZHU, Qinghua; SARKIS, Joseph. The moderating effects of institutional pressures on emergent green supply chain practices and performance. International Journal of Production Research, v. 45, n. 18-19, p. 4333-4355, 2007.

ZUCKER, Lynne G. The role of institutionalization in cultural persistence. American Sociological Review, v. 42, n. 5, p. 726-743, 1977.

Regina Célia Nazar Fialho é doutora em administração pelo Centro de Pós-Graduação e Pesquisa em Administração (Cepead/UFMG). Professora do IBMEC/Administração (Rio de Janeiro, RJ). E-mail: reginanfialho@gmail.com.

Ricardo S. Martins é doutor em economia aplicada, professor em gestão de operações e logística no Centro de Pós-Graduação e Pesquisa em Administração (Cepead/UFMG). Pesquisador do Núcleo Interdisciplinar de Pesquisas, Ensino e Extensão em Logística da UFMG (Nipe-LOG/UFMG). E-mail: rmartins@ face.ufmg.br. 


\section{Apêndice}

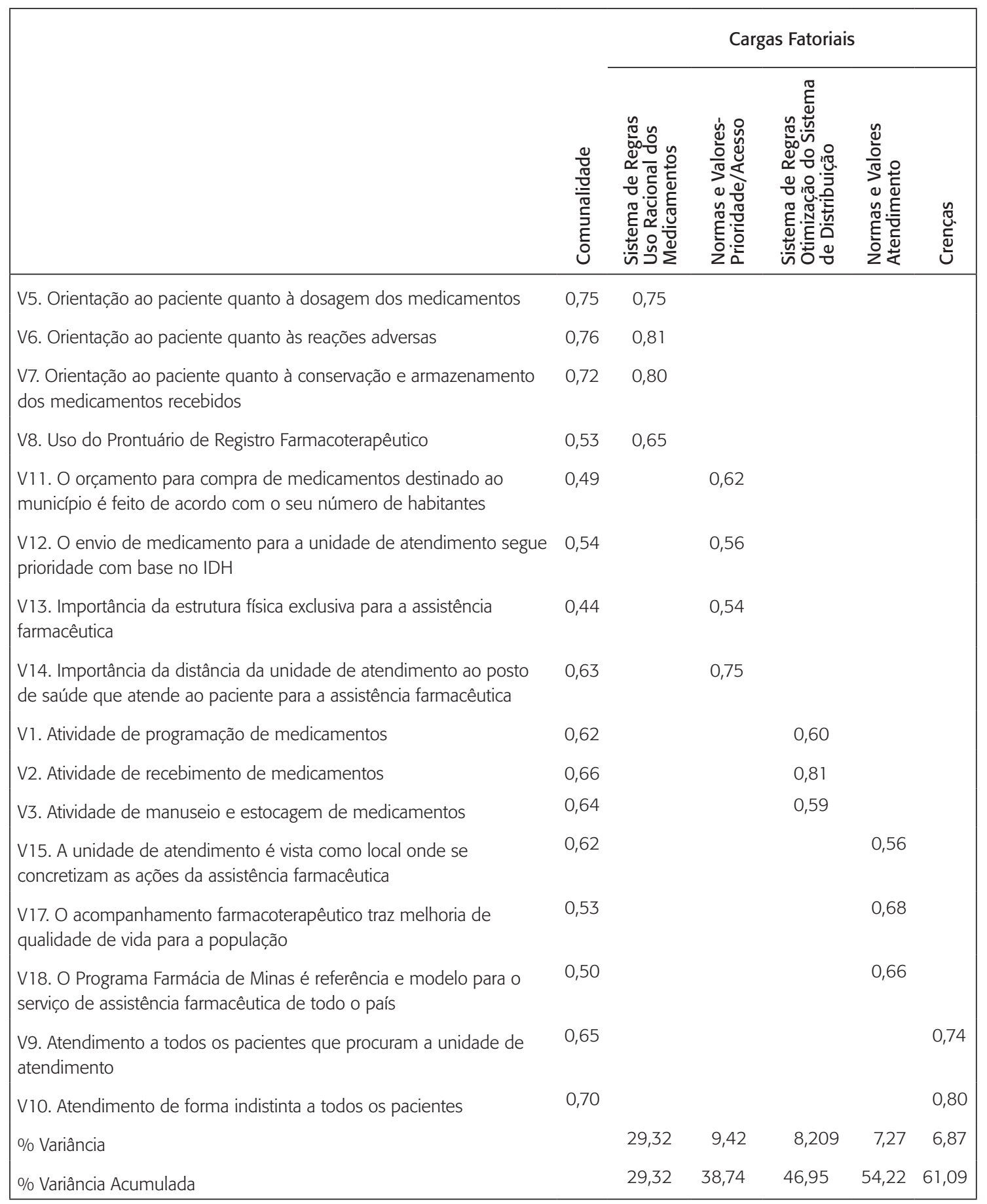




\begin{tabular}{|c|c|c|c|c|c|c|c|}
\hline Construtos & 1.1 . & 1.2 . & 2.1 & 2.2 & 3. & 4. & 5. \\
\hline $\begin{array}{l}1.1 \text { Sistema de Regras - Uso Racional dos } \\
\text { Medicamentos }\end{array}$ & 0,68 & & & & & & \\
\hline $\begin{array}{l}\text { 1.2 Sistema de Regras - Otimização do Sistema de } \\
\text { Distribuição }\end{array}$ & 0,11 & 0,57 & & & & & \\
\hline 2.1 Normas e Valores - Prioridade e Acesso & 0,07 & 0,00 & 0,58 & & & & \\
\hline 2.2 Normas e Valores - Atendimento & 0,01 & 0,03 & 0,02 & 0,63 & & & \\
\hline 3. Crenças & 0,14 & 0,01 & 0,11 & 0,00 & 0,56 & & \\
\hline 4. Desenho da Rede & 0,03 & 0,16 & 0,00 & 0,00 & 0,01 & - & \\
\hline 5. Desempenho & 0,07 & 0,18 & 0,00 & 0,00 & 0,00 & 0,12 & - \\
\hline Construtos & & & $\begin{array}{l}\text { iabilid } \\
\text { mpost }\end{array}$ & & $\begin{array}{l}\text { Alfa de } \\
\text { Cronbach }\end{array}$ & & AVE \\
\hline 1.1 Sistema de Regras - Uso Racional dos Medicamer & & & 0,89 & & 0,86 & & 0,68 \\
\hline 1.2 Sistema de Regras - Otimização do Sistema de Dis & buição & & 0,80 & & 0,63 & & 0,57 \\
\hline 2.1 Normas e Valores - Prioridade e Acesso & & & 0,78 & & 0,63 & & 0,58 \\
\hline 2.2 Normas e Valores - Atendimento & & & 0,77 & & 0,72 & & 0,63 \\
\hline 3. Crenças & & & 0,72 & & 0,62 & & 0,56 \\
\hline
\end{tabular}




\begin{tabular}{|c|c|c|}
\hline Construto & Variáveis & VIF \\
\hline \multirow[t]{6}{*}{ Desenho da Rede } & V19. Distância em km do armazém central até cada unidade de atendimento & 1,02 \\
\hline & V20. Quantidade máxima de medicamentos solicitada por cada unidade de atendimento & 1,08 \\
\hline & $\begin{array}{l}\text { V21. Nível de estoques adequados de forma a atender à demanda da unidade de } \\
\text { atendimento }\end{array}$ & 1,05 \\
\hline & V22. Quantidade de volumes transportados, por pedido, para cada unidade de atendimento & 1,30 \\
\hline & V23. Nível de utilidade das informações de demanda e estoques do Sigaf & 1,06 \\
\hline & $\begin{array}{l}\text { V24. Número de fornecedores necessários para suprir os medicamentos solicitados pelas } \\
\text { unidades de atendimento, por entrega }\end{array}$ & 1,26 \\
\hline \multirow[t]{6}{*}{ Desempenho } & V25. Tempo médio de recebimento de pedidos solicitados, por unidade de atendimento & 1,12 \\
\hline & $\begin{array}{l}\text { V26. Percentual de uso da capacidade instalada para estocar medicamento, por unidade de } \\
\text { atendimento }\end{array}$ & 1,04 \\
\hline & V27. Tempo, em média, em que a unidade de atendimento tem ruptura de estoque & 1,02 \\
\hline & $\begin{array}{l}\text { V28. Custo médio de transporte de medicamentos solicitados pela unidade de atendimento, } \\
\text { por pedido }\end{array}$ & 1,04 \\
\hline & V29. Percentual da exatidão das informações de demanda e de estoque geradas pelo Sigaf & 1,07 \\
\hline & $\begin{array}{l}\text { V30. Percentual de medicamentos faltantes, por entrega, tendo em vista o atraso de entrega } \\
\text { do fornecedor no armazém central }\end{array}$ & 1,05 \\
\hline
\end{tabular}

